\title{
First Principles Study of Structural, Electronic and Magnetic Properties of Defected (Monovacant) Hexagonal Boron Nitride Sheet
}

\section{R. Karki, K. Khatri, K. Adhikari, N. P. Adhikari and N. Pantha}

Journal of Nepal Physical Society

Volume 7, Issue 4, December 2021

ISSN: 2392-473X (Print), 2738-9537 (Online)

Editors:

Dr. Binod Adhikari

Dr. Bhawani Joshi

Dr. Manoj Kumar Yadav

Dr. Krishna Rai

Dr. Rajendra Prasad Adhikari

Mr. Kiran Pudasainee

JNPS, 7 (4), 19-27 (2021)

DOI: http://doi.org/10.3126/jnphyssoc.v7i4.42927

Published by:

Nepal Physical Society

P.O. Box: 2934

Tri-Chandra Campus

Kathmandu, Nepal

Email: nps.editor@gmail.com

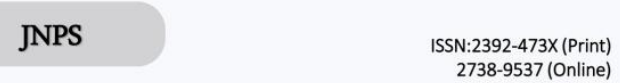

Journal of

Nepal Physical Society

Volume 7, Issue 4, December 2021

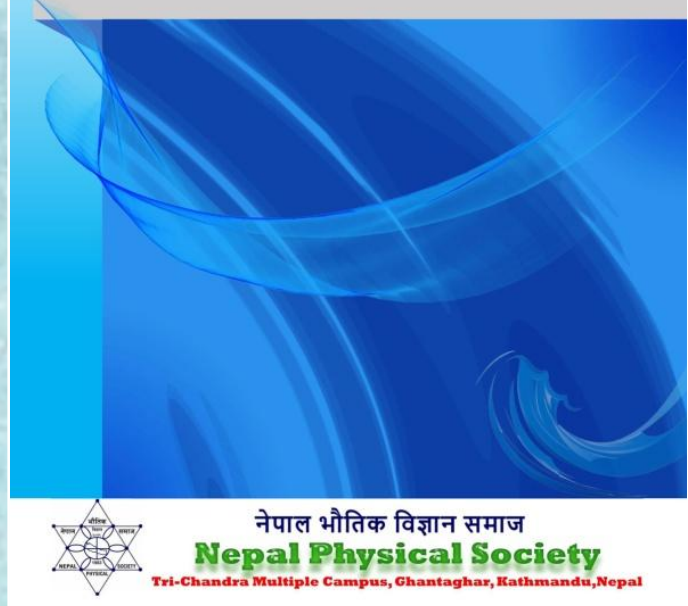




\title{
First Principles Study of Structural, Electronic and Magnetic Properties of Defected (Monovacant) Hexagonal Boron Nitride Sheet
}

\author{
R. Karki ${ }^{1,3}$, K. Khatri ${ }^{1}$, K. Adhikari ${ }^{2,3}$, N. P. Adhikari ${ }^{1}$ and N. Pantha ${ }^{1, ~ *}$ \\ ${ }^{1}$ Central Department of Physics, Tribhuvan University, Kirtipur, Kathmandu, Nepal \\ ${ }^{2}$ Gandaki University, Pokhara, Nepal \\ ${ }^{3}$ Department of Physics, Prithvi Narayan Campus, Pokhara \\ *Corresponding Email: mrnurapati@gmail.com
}

Received: 18th November, 2021; Revised: 17th December, 2021; Accepted: 28th December, 2021

\begin{abstract}
The structural, electronic, and magnetic properties of vacancy structures with triangular shape are related to the defect in single hexagonal boron nitride (h-BN) sheet. It is investigated by using density functional theory calculations. The first-principles calculations based GGA functionals have been implemented to study the structural, electronic and magnetic properties of pure and defected hexagonal boron nitride (h-BN) monolayer sheet using Quantum ESPRESSO (QE) package, 6.5 version. The calculated values of formation energy reveal the structural stability of the defected systems. The formation energies for $\mathrm{B}$ and $\mathrm{N}$ vacant system are found to be $16.45 \mathrm{eV}$ and $12.87 \mathrm{eV}$ respectively. This predicts that the $\mathrm{N}$ vacant system is more preferable with lower formation energy. The defect in h-BN seems to be changing its band gap and magnetic properties. The $6.25 \% \mathrm{~B}$-vacancy results h-BN to be half metallic ferromagnetic with total magnetization of $2.74 \mu \mathrm{B} /$ cell. Further, $6.25 \% \mathrm{~N}$-vacancy causes it to be magnetic semiconductor with total magnetization of $1.00 \mu \mathrm{B} /$ cell.
\end{abstract}

Keywords: Formation energy, Vacancies, Band structure, Density of states.

\section{INTRODUCTION}

The study of crystalline materials has been carried out since long. Discovery of graphene in 2004 and its astonishing properties have given birth to a new class of materials known as two dimensional (2D) materials, like phosphorene, hexagonal boron nitride, silicene, stanene, transition metal di-chalcogenides (TMDCs) etc. [1] Intense studies in such materials are growing these days. In our work, we have studied different properties of defected (monovacant) two dimensional hexagonal boron nitride.

Crystal defect is one of the imperfections in the regular geometrical arrangement of the atoms in a crystalline solid. One of the defects is missing of atom/s from its normal position, is called vacancy [2]. Vacancy is a type of point defect and is essential for a crystalline solid to be in thermal equilibrium [3, 4]. As similar to bulk crystals, we observe various changes due to vacancy defect and addition of impurity atoms, which lead to point defect in a monolayer crystal. [5]
Boron nitride is a thermal and chemical resistant refractory compound of boron and nitrogen with the chemical formula BN. It is a modern manmade compound that perfectly parallels to the crystalline structure and properties of naturally occurring elementary carbon atoms. [6] It has potential use in nanotechnology. [7] The structural analogue of a carbon nanotube (CNT), a BN nanotube (BNNT) was first predicted in 1994; since then, it has become one of the most intriguing non-carbon nanotubes. [8] Hexagonal boron nitride, cubic boron nitride, amorphous boron nitride and wurtzite (zinc and iron sulfide mineral) forms of boron nitride are different crystalline forms of $\mathrm{BN}$ which are isoelectronic to a similarly structured carbon lattice. Hexagonal boron nitride is one of the known representative crystal structures of BN. [9]

Hexagonal boron nitride was first discovered in 2005. [10] It has similar bonding and structure to monolayer graphite. It is comprised of alternating 
boron and nitrogen atoms in a honeycomb arrangement, consisting of sp2-bonded twodimensional layers in which boron and nitrogen atoms are bounded by strong covalent bonds within each layer of hexagonal $\mathrm{BN}$ whereas the layers are held together by weak van der Waals' forces [11] (figure 1). Although hexagonal boron nitride has a similar two-dimensional structure, it has distinct chemical species from graphene.[12] In order to clarify the lattice defects in h-BN such as vacancies or edges, the individual boron and nitrogen atoms should be directly imaged and even distinguished otherwise precise defect structures cannot be deduced. [13] The monolayer h-BN has an atomically flat, a very low roughness and no dangling bonds on its surface. Its cell parameters and other properties are described in previous studies. [14, 15] The allotropes of h-BN show different physical/electronic properties and thus carry various applications; in deep ultraviolet (DUV) photo electronic devices, micro and nanodevices and so on. [16, 17]

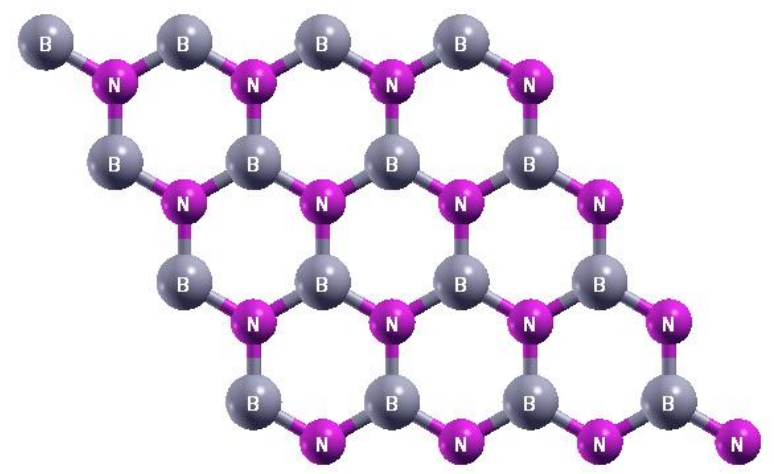

Fig. 1: A monolayer hexagonal boron nitride (h-BN) structure where $\mathrm{B}(\mathrm{N})$ represents boron (nitrogen) atom. The optimized unit cell structure was replicated in $\mathrm{x}$ and y directions to get this $(4 \times 4)$ supercell.

The monolayer h-BN is a wide gap insulator and can be tuned as per requirement. Also the band gap and its nature is not uniquely described. It precisely depends on the number of techniques employed for its determination. [18] There are some techniques that give the direct band gap and others predict its indirect band gap. The magnitude has been reported in the range of 3.0 to $7.5 \mathrm{eV}$. [19, 20] Hence, it could be useful as a complementary twodimensional dielectric substrate for graphene electronics. [21] It is chemically/thermally stable and possesses Young's modulus in between 0.71 to $0.97 \mathrm{TPa}$. [22]
After this introduction section, the paper incorporates computational details in section 2, results and discussion in chapter 3, conclusions in chapter 4 before future scopes and references.

\section{COMPUTATIONAL DETAILS}

First principles calculations have been performed using DFT calculations with GGA for exchangecorrelation energy implemented in QuantumESPRESSO package to study the stability, geometrical structures, electronic and magnetic properties of pristine hexagonal boron nitride (h$\mathrm{BN}$ ) as well as mono-vacant (Boron and Nitrogen vacancies) h-BN system in two dimensions (2D). We have used computational methods as per facilities available with Quantum ESPRESSO simulation package. It is an integrated suite of computer codes for electronic-structure calculations and materials modeling, based on densityfunctional theory, plane waves, and pseudopotentials. [23] To visualize our molecular and the crystalline structures (figure 2), we have used XCrysDen and VESTA as required softwares.

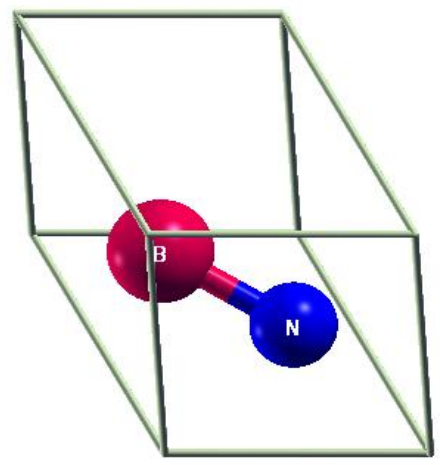

Fig. 2: Optimized primitive unit cell, constructed by using XcrysDen software.

We have implemented spin polarized density functional theory to perform up spin and down spin calculations separately. The convergence tests for ecutwfc, lattice parameter ' $a$ ' and K-points have been performed to establish the suitable environment before optimizing the most stable structures for our calculations. The samples of pure $\mathrm{h}-\mathrm{BN}$ sheet and single vacant (B and $\mathrm{N}$ sites) h-BN sheets are then considered for further details.

\section{RESULTS AND DISCUSSION}

We hereby discuss the structural, electronic and the magnetic properties of the defected systems with reference to pure h-BN. 
3.1 Structural properties of pure and defected hexagonal boron nitride ( $h-B N)$

The $(4 \times 4)$ supercell of monolayer h-BN (assumed unit cell hereby) was allowed to relax using BFGS quasi-newton algorithm. [24] We found very little change in the atomic position after relaxation and thus found very small variations in bond lengths and bond angles.

The relaxed primitive cell structure gives lattice parameter of $\mathrm{a}=\mathrm{b}=2.51 \AA$. Also the $\mathrm{B}-\mathrm{N}$ bond lengths, N-B-N/B-N-B bond angles and the lattice parameters resemble with the reported values. [15] The interlayer

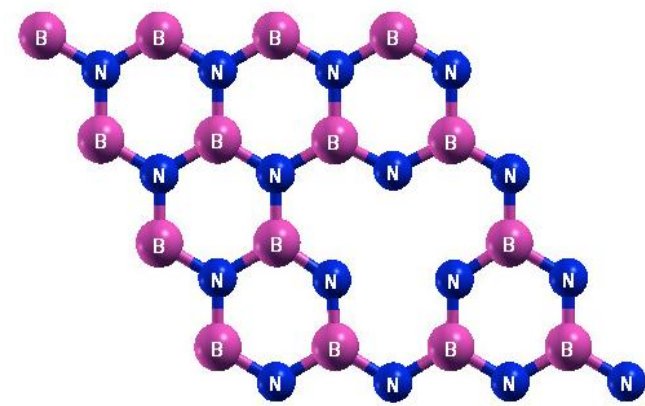

Fig. 3: Single boron vacant site of $\mathrm{h}-\mathrm{BN}$ after relaxation. separation was kept at $20 \AA$ ( $c=20.02 \AA)$ to avoid the interaction between the layer to layer periodic images. After obtaining the desired structure and geometry of pure h-BN, we created a single vacancy in the supercell by removing one boron and one nitrogen atom separately so that atoms in a unit cell are decreased by one for each vacant system (each of $\mathrm{B} / \mathrm{N}$ vacancy $=6.25 \%$ ) (figures 3 and 4 ). Now the unit cell contains 31 atoms with the basis of 2 atomic species. The vacant systems (vacancy at $B$ site: $V_{B}$ and vacancy at $\mathrm{N}$ site $\mathrm{V}_{\mathrm{N}}$ ) are then relaxed to get their ground states.

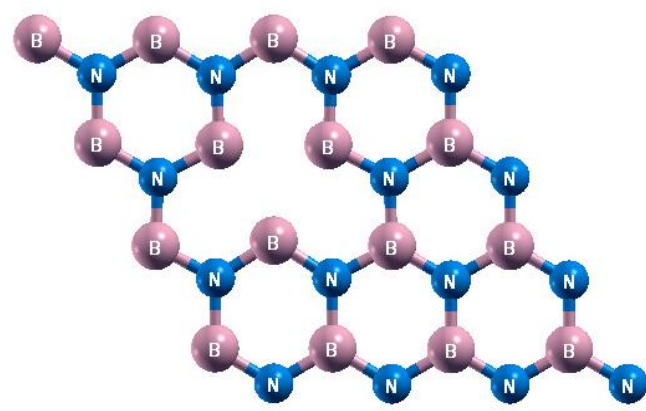

Fig. 4: Single nitrogen vacant site of $h-B N$ after relaxation.

Table 1: Structural parameters of $h-B N$ sheet with boron vacancy $\left(V_{B}\right)$

\begin{tabular}{l|l}
\hline \multicolumn{2}{c}{ Structural detail of $\mathbf{4} \times \mathbf{4} \mathbf{h}-\mathbf{B N}$ sheet with $\mathbf{V}_{\mathbf{B}}$} \\
\hline N-B-N bond angles & $123.94^{\circ}, 123.94^{\circ}, 123.94^{\circ}$ \\
Nearest neighbor B-N-B bond angles & $118.23^{\circ}, 118.23^{\circ}, 118.23^{\circ}$ \\
Nearest neighbor B-N bond lengths towards $\mathrm{V}_{\mathrm{B}}$ & $1.41 \AA$ \\
\hline
\end{tabular}

Table 2: Structural parameters of the $h-B N$ with nitrogen vacancy $\left(V_{N}\right)$

\begin{tabular}{l|l}
\hline \multicolumn{2}{c}{ Structural detail of $\mathbf{4} \times \mathbf{4} \mathbf{h}-\mathbf{B N}$ sheet with $\mathbf{V}_{\mathbf{N}}$} \\
\hline B-N-B bond angles & $119.7^{\circ}, 119.71^{\circ}, 119.7^{\circ}$ \\
Nearest neighbor N-B-N bond angles & $115.72^{\circ}, 115.72^{\circ}, 115.72^{\circ}$ \\
Nearest neighbor B-N bond lengths towards $\mathrm{V}_{\mathrm{N}}$ & $1.46 \AA$ \\
\hline
\end{tabular}

When we create a vacancy on pure h-BN by removing a boron or a nitrogen atom, several changes were observed. Both the systems show triangular vacancies (void space) at the center of the samples which were usually occupied by a $\mathrm{B} / \mathrm{N}$ atom. However, the boron vacancy $\left(\mathrm{V}_{\mathrm{B}}\right)$ and nitrogen vacancy $\left(\mathrm{V}_{\mathrm{N}}\right)$ have opposite orientation. This resembles with the previous studies. $[18,25]$ Because of absence of one of the atoms in usual triangular bonding, interatomic strength in between nearby atoms changes. The changes can be observed in the tables (1) and (2).

For boron site vacancy (table 1) N-B-N bond angles are increased $\left(123.94^{\circ}\right)$ with reference to the usual triangular angles $\left(120^{\circ}\right)$. However, B-N-B bond angles are decreased (by $1.77^{\circ}$ ) and become $118.23^{\circ}$. The N-B bond length is decreased (to $1.41 \AA$ ) with reference to that in pure h-BN (1.44 $\AA$ in our calculation). Because of dangling bonds at nitrogen atoms (due to absence of nearby B atom), they seem to be attracted towards 
the other nearby bonded B atoms and get squeezed. Table 2 shows the geometrical parameters in nitrogen vacancy defect $\left(\mathrm{V}_{\mathrm{N}}\right)$. As similar to boron vacancy, NB-N and B-N-B get different values in nitrogen site vacancies. Dissimilar to $\left(\mathrm{V}_{\mathrm{B}}\right)$, average of angle $\mathrm{B}-\mathrm{N}-\mathrm{B}$ $\left(119.7^{\circ}\right)$ is greater than that of N-B-N (115.72). However, the similarity in both the cases is that angle which centers to nearby atoms of the missing atom are larger in comparison to their neighbors. It could be due to pulling of the atom away from its normal position which otherwise bonded with the missing atom. The B-N bond length in non-defective region is found to be $1.43 \AA$ which is almost equal to that in calculated pristine $\mathrm{h}-\mathrm{BN}$ and the experimental value (1.44 $\AA$ ) within the error of $0.1 \AA$. [25]

\subsection{Energy and Stability}

The values of the total and formation energies predict the stability of the sample. We calculate the total energy, the Fermi energy, the Kohn-Sham states, the energy Eigen values and the magnetic moment of our system to explain the magnetization along with the forces and stresses on individual atoms. With these data, we calculate the defect formation energy for vacant system in the h-BN sheet. The formula for the defect formation energy $\left(\mathbf{E}_{\mathrm{F}}\right)$ is,

$$
\mathbf{E}_{\mathrm{F}}=\left(\mathbf{E}_{\mathrm{d}}-\mathbf{E}_{\mathrm{p}}\right)+\mathbf{n} \boldsymbol{\mu} .
$$

In this equation, $\mathbf{E}_{\mathrm{d}}$ is the total energy of defected (single vacant) h-BN sheet, Ep is total energy of pristine h-BN sheet, $\mathbf{n}$ is the number of boron or nitrogen atoms removed and $\boldsymbol{\mu}$ is the chemical potential of the atom removed to create the vacancy. $[26,27]$ The chemical potential $\boldsymbol{\mu}$ for $\mathrm{B}$ and $\mathrm{N}$ atoms was calculated using an isolated system of single atom. The calculated values are shown in table 3 .

Table 3: Different energy values required to calculate the formation energy (in Rydberg; 1 Rydberg = 13.6 eV)

\begin{tabular}{|c|c|c|c|}
\hline \multicolumn{2}{|c|}{ Formation energy of single B vacant $h-B N$} & \multicolumn{2}{|c|}{ Formation energy of single Nitrogen vacant $h-B N$} \\
\hline Study in system & $\operatorname{Energy}\left(\mathrm{R}_{\mathrm{y}}\right)$ & Study in system & $\operatorname{Energy}\left(\mathrm{R}_{\mathrm{y}}\right)$ \\
\hline Single Boron vacant h-BN $\left(E_{d}\right)$ & -625.613 & Single Boron vacant $\mathrm{h}-\mathrm{BN}\left(\mathrm{E}_{\mathrm{d}}\right)$ & -609.029 \\
\hline Pristine $4 \times 4$ h-BN $\left(E_{p}\right)$ & -637.809 & Pristine $4 \times 4$ h-BN $\left(E_{p}\right)$ & -637.81 \\
\hline Energy of isolated B atom $(\mu)$ & -10.986 & Energy of isolated B atom $(\mu)$ & -27.835 \\
\hline Formation energy & 1.21 & Formation energy & 0.946 \\
\hline
\end{tabular}

Table 3 shows formation energy at B site vacancy $\left(\mathrm{V}_{\mathrm{B}}\right)$ as $1.21 \mathrm{Ry}(16.45 \mathrm{eV})$ and that at $\mathrm{N}$ site vacancy $\left(\mathrm{V}_{\mathrm{N}}\right) 0.946 \mathrm{Ry}(12.87 \mathrm{eV})$. The positive values of formation energy in the table indicate that the energy should be given to form such vacant systems. Higher the formation energy means more the energy is to be given to form such a vacancy, and less preferable to construct to construct. In our calculation, $V_{B}$ has higher formation energy and is a less favorable vacant system.

\subsection{Band Structure Calculation}

An isolated atom has discrete energy levels and the electrons are filled on that levels in accordance with Pauli Exclusion Principle if there is no any kind of perturbation. These discrete energy levels are called atomic orbitals. However, these discrete energy levels can be perturbed by bringing a large number of atoms together. In the presence of any other atoms near to the initial atom under consideration, each energy level gets split into a pair of energy levels with narrow width. Consequently, when we bring large number of atoms close together to form a molecule, their atomic orbital split into a large number of closely packed discrete molecular orbitals. In solids, the energy levels of electrons form continuous bands of energy rather than the discrete energy levels of the atoms in isolation. The continuum level of energy is called an energy band.

The energy bands can be empty, filled, forbidden or mixed. [28] A solid is called an insulator if the allowed energy bands are either completely filled or empty. Metals are those whose bands are partly filled whereas the semiconductors have one or two bands slightly filled or slightly empty with band width of nearly $1 \mathrm{eV}$. [29] By using an irreducible part of the first Brillouin zone, we have calculated the entire band structure of our system. The first Brillouin zone of the hexagonal lattice with $\Gamma-\mathrm{K}-\mathrm{M}$ $\Gamma$ high symmetry path has been considered. The structure is depicted in figure 5. 


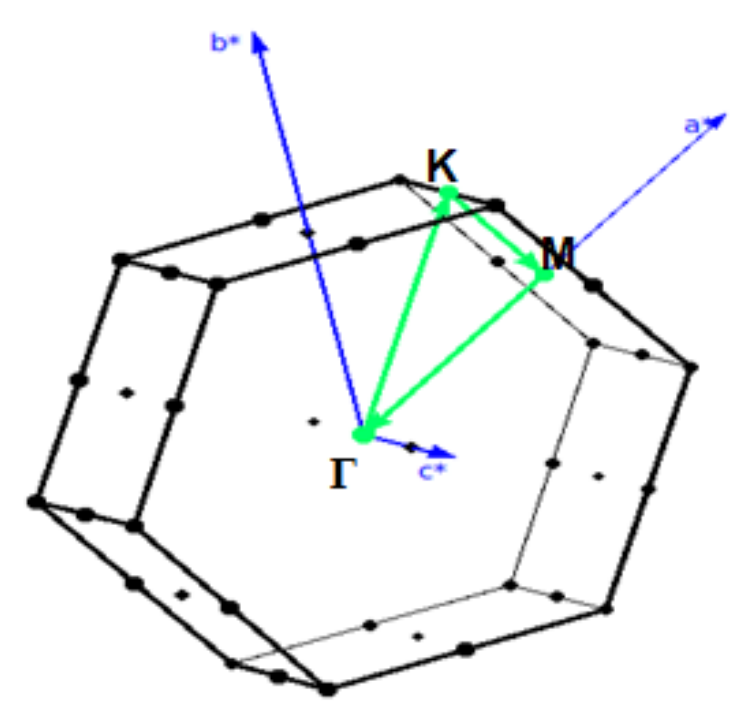

Fig. 5: First Brillouin zone of a hexagonal lattice with high symmetric points. We have chosen $\Gamma-\mathrm{M}-\mathrm{K}-\Gamma$ path.

\subsection{Band Structure of Pure Hexagonal Boron Nitride}

Previous studies show that the pristine h-BN is a non-magnetic insulator with large band gap. [30] The basis of two atoms (boron and nitrogen) in a primitive cell of $\mathrm{h}$-BN offers eight valence electrons and supports the idea of its insulating nature. However, the nature of band gap (direct or indirect) is a matter of discussion as different

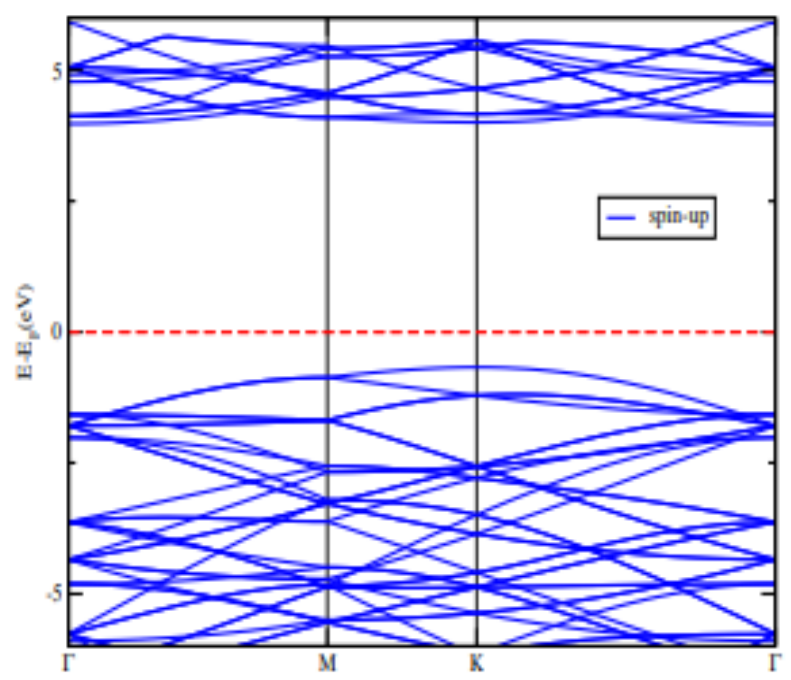

methods predict different results. This could be due to flat type of bands which have nearly equal values of energies at different $\mathrm{K}$ points. Here, we consider the primitive pristine $\mathrm{h}-\mathrm{BN}$ and that of size $4 \times 4$ supercell to validate our results with them. Our study shows a direct gap at K-point with the magnitude of $4.64 \mathrm{eV}$ (figure 6). Further, the spinpolarized calculations obtain the identical band structures for up and down-spin electrons (figure 7). The results are in good agreement with the previous study. [31]

The value of Fermi energy is found to be $-4.0162 \mathrm{eV}$ and set to zero in figures (figures 6 and 7) for its proper visualization.

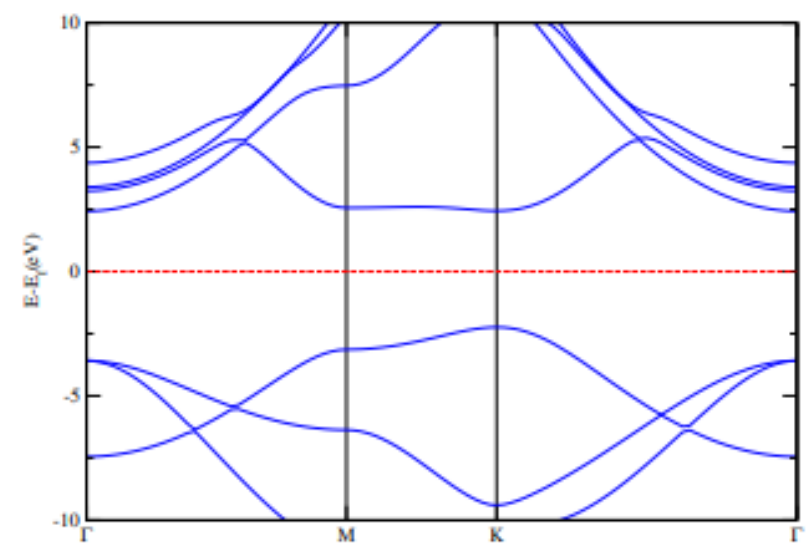

Fig. 6: Band structure of pristine h-BN using primitive cell

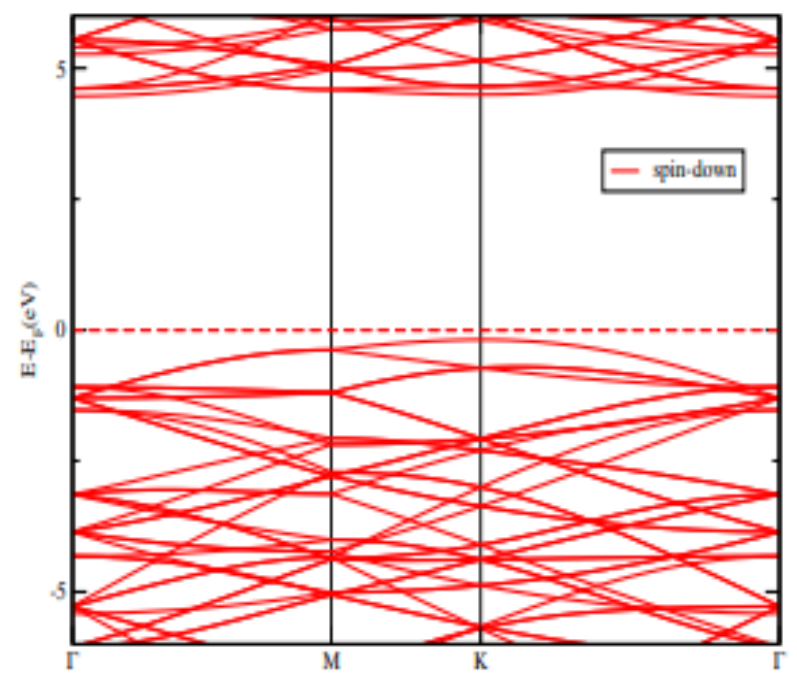

Fig. 7: Spin polarized calculations for band structures of pristine h-BN using $4 \times 4$ supercell $\left(\right.$ Fermi energy $E_{F}=-4.016$ $\mathrm{eV}$ is set to zero for clarity).

\subsection{Density of States and Magnetization}

The non-magnetic nature of pristine h-BN can be seen from the spin polarized density of states plot.
Distribution of available states for both up and down spins are symmetrical (figure 8 and 9) which reveals that the monolayer $\mathrm{h}-\mathrm{BN}$ is nonmagnetic. 


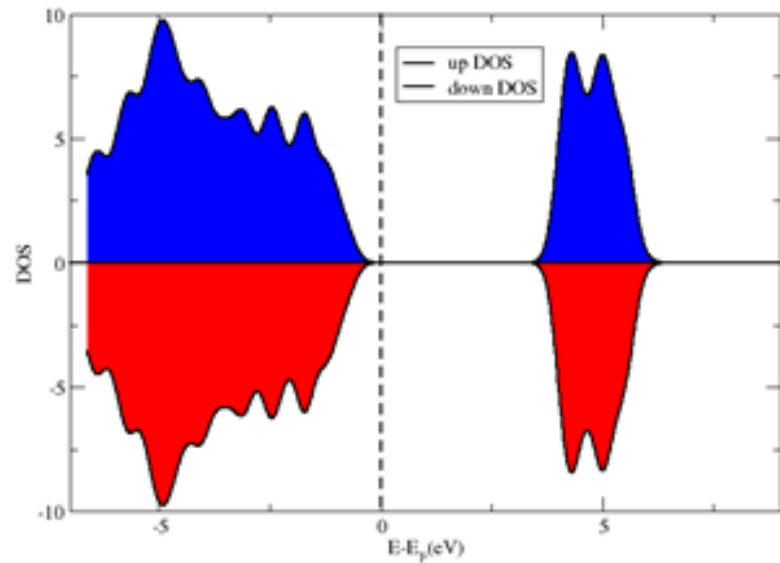

Fig. 8: Density of states for $4 \times 4$ supercell of pristine h-BN.

Projected DOS shows the contribution of each of the electronic orbitals (which are supposed to be the valence electrons in respective atomic pseudo potentials). It remains high in the region where many orbitals are accumulated and low in the regions where the number of orbitals are less in number. Also the DOS is completely zero in the region where there are no electronic orbitals i.e. between the valance and the conduction bands. Below Fermi level, bands are mainly dominated by $2 p$ orbitals of $\mathrm{N}$ atom and above Fermi level by $2 \mathrm{p}$ orbitals of B atom. In totality, 'p' orbitals are dominant over the 's' orbitals.

\subsection{Electronic and Magnetic properties of mono- vacant $h-B N$}

Vacancy in any material changes the electronic as well as magnetic properties. We observe such changes in vacant systems using spin polarized

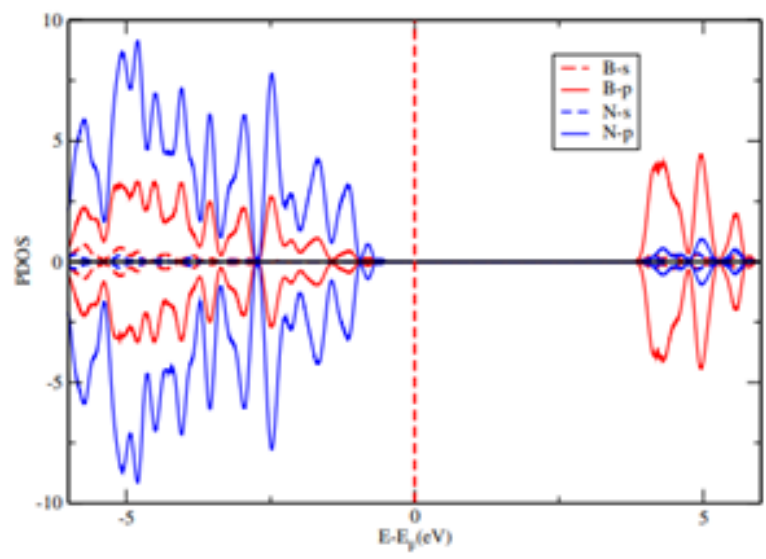

Fig. 9: PDOS for $s$ and $p$ orbitals of $B$ and $N$ atoms of $4 \times 4$ supercell of pristine h-BN.

SCF, DOS and projected DOS calculations as similar to previous studies of related systems. [32]

\subsubsection{Single Boron vacant $4 \times 4 \mathrm{~h}-\mathrm{BN}$ sheet $\left(V_{B}\right)$}

Figures 10 shows the band structure of a single vacant $h-B N$ due to $V_{B}$. In the figure, we observe that down spin electrons in valance band cross the Fermi level and also go above the Fermi level (as similar to that in metals). Here, we can see only few states and the energy levels of valance orbitals (s and $\mathrm{p}$ ) of $\mathrm{B}$ and $\mathrm{N}$ are changed around the Fermi level. Since the down spin electrons show the metallic nature and the up spin electrons show insulating nature, the system under consideration seems to be half metallic.

In figure 10 , we see that vacancy defect specially influences near to Fermi level. The Fermi level is shifted down towards the occupied state as compared to defect-free h-BN.
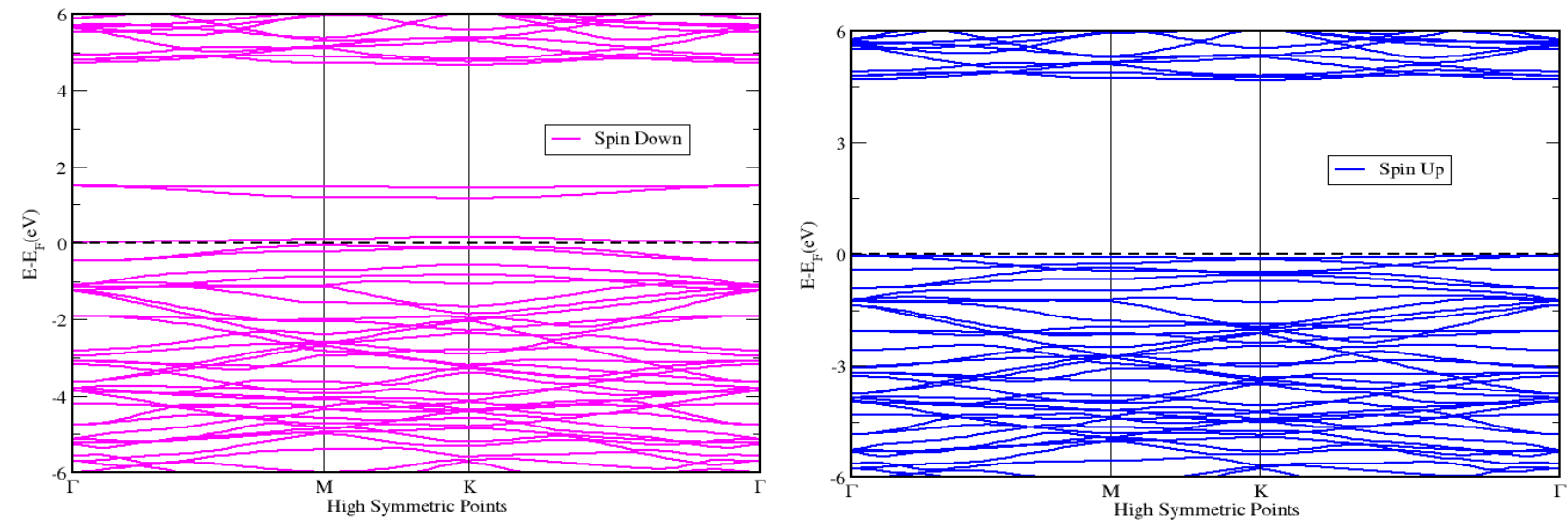

Fig. 10: Down and up spin band structures of single boron vacant h-BN $\left(\mathrm{V}_{\mathrm{B}}\right)$ [Fermi energy, $E_{F}=-4.169 \mathrm{eV}$ is set to zero]. 


\subsubsection{Density of States and Magnetization of $V_{B}$}

Dissimilar to the pristine h-BN, defected $\left(\mathrm{V}_{\mathrm{B}}\right)$ h-BN shows the different distribution of available states for up and down spins (figures 11, 12). The asymmetrical up and down spin orbitals signify magnetic nature of the mono-vacant layer. Our calculations find the magnitude of total magnetization as $2.74 \mu_{B}$ for $V_{B}$. The isolated $B$ and $\mathrm{N}$ atoms offer magnetism as they have magnetic moment of $1 \mu_{\mathrm{B}}$ and $3 \mu_{\mathrm{B}}$ respectively. However, in their combined structure, total magnetization is different than their sum. It could be due to hybridization in between the atomic orbitals which promotes redistribution of electronic charge. The projected DOS (figure 12) monitors the contribution of atomic orbitals of each atom/s.

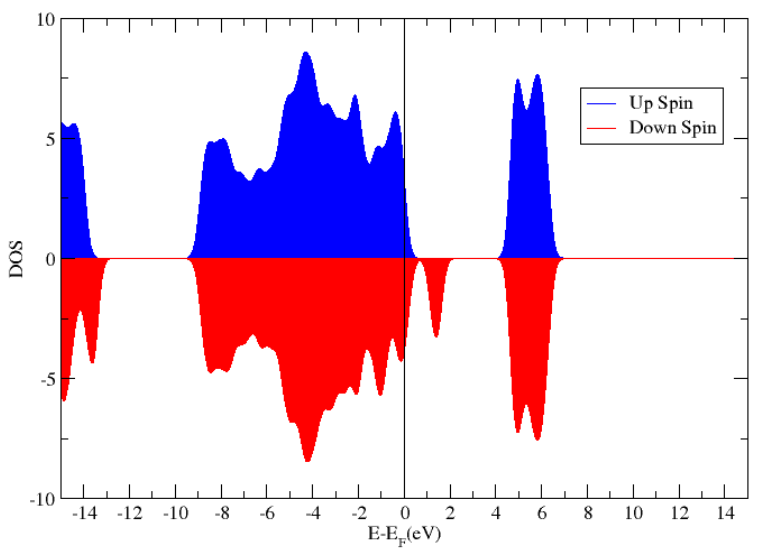

Fig 11: Density of states plot for $\left(\mathrm{V}_{\mathrm{B}}\right)$

The projected DOS calculations show that the available states around Fermi level are large due to $\mathrm{B}$ and $\mathrm{N}$ atoms. In both $\mathrm{B}$ and $\mathrm{N}$ atoms, $2 \mathrm{p}$ orbitals are dominant over the $\mathrm{s}$ orbitals. Hence, the contribution of $2 p$ orbitals of $\mathrm{B}$ and $\mathrm{N}$ atoms (for magnetization) is relatively greater than $1 \mathrm{~s}$ orbital.

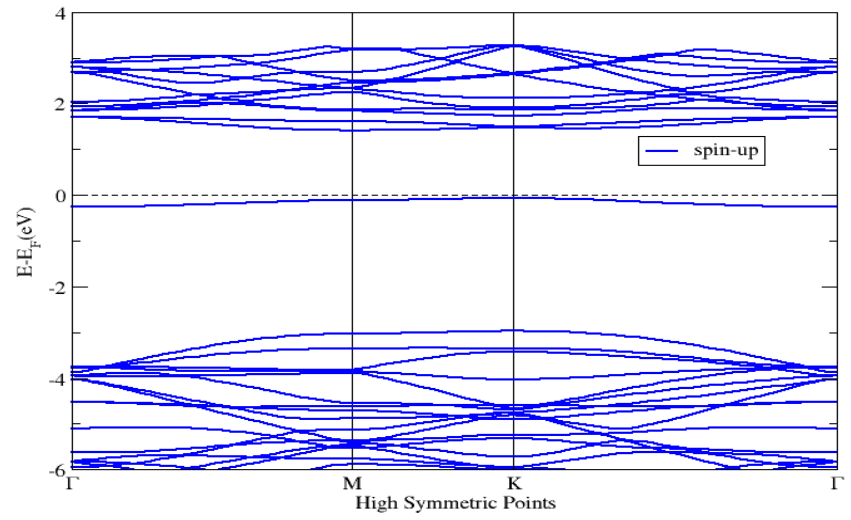

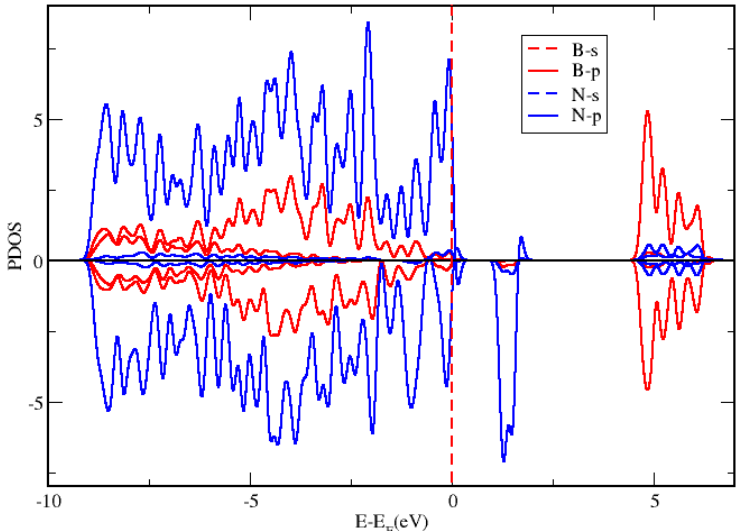

Fig. 12: Projected Density of States of h-BN with $V_{B}$

\subsubsection{Single nitrogen vacancy in $4 \times 4 \mathrm{~h}-\mathrm{BN}$ sheet $\left(\mathrm{V}_{\mathrm{N}}\right)$}

Figure 13 (at left and right) shows the band structure of nitrogen site of vacant h-BN. The up spin band structure reveals the fact that electrons in valance band lie $0.08 \mathrm{eV}$ below the Fermi level whereas for down spin electrons, conduction band lies $0.33 \mathrm{eV}$ above the Fermi level. Hence the total band gap near to Fermi level (in between valence and conduction band) becomes $0.41 \mathrm{eV}$. The value falls in the regime of semiconductor and concludes that wide gap (insulator) pristine h-BN changes to semiconductor upon creation of $\mathrm{N}$-site vacancy.

The new states present in the band structure of $\mathrm{V}_{\mathrm{N}}$, especially near to Fermi level are the effect of Nsite vacancy. The minute analysis shows that energy levels of valance orbitals ( $\mathrm{s}$ and $\mathrm{p}$ ) of B and $\mathrm{N}$ are also changed around the Fermi level. The independent pictures in figure 13 inform that the system behaves as semiconductor for up spin (1.44 $\mathrm{eV}$ ) and insulator for down spin $(3.27 \mathrm{eV})$. Since these gaps observed at $\mathrm{K}$ and $\mathrm{M}$ points respectively, the $\mathrm{N}$-site vacant $\mathrm{h}$-BN possesses indirect type of energy gap nearby Fermi level.

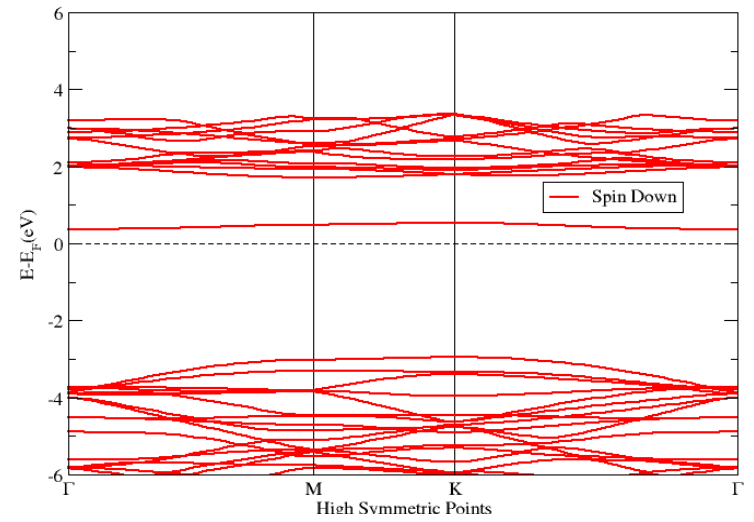

Fig. 13: Up and down spin band structures of single nitrogen vacant $h-B N\left(V_{N}\right)$, [Fermi energy $E_{F}=-1.379$ eV is set to zero for the clarity of the picture]. 
Our calculations found that the Fermi level shifts up (by $2.637 \mathrm{eV}$ ) in $\mathrm{V}_{\mathrm{N}}$ with reference to pure h$\mathrm{BN}$. Also the vacant system gets magnetism (1 $\mu \mathrm{B} /$ cell) upon removal of $\mathrm{N}$ atom in pure $\mathrm{h}-\mathrm{BN}$.

\section{CONCLUSIONS}

We have studied the effect of single atom vacancy defects (at boron and nitrogen sites separately which accounts for $6.25 \%$ of the respective atoms) in monolayer hexagonal boron nitride (h-BN) by using density functional theory level of approximations in Quantum ESPRESSO codes. Both the defected systems, $V_{B}$ (vacancy at $B$ site) and $\mathrm{V}_{\mathrm{N}}$ (vacancy at $\mathrm{N}$ site), show different geometrical, electronic and magnetic properties with reference to the pristine h-BN. In geometry, both the systems show triangular vacancies (void space) at the center of the samples which used to be occupied by boron or nitrogen atom in pristine structure. However, the boron vacancy $\left(\mathrm{V}_{\mathrm{B}}\right)$ and nitrogen vacancy $\left(\mathrm{V}_{\mathrm{N}}\right)$ have opposite orientations. The bond angles and bond lengths nearby vacancy get changed due to the presence of unsaturated bonds nearby of it. The vacancy formation energy, the energy required to form a vacancy by removing an atom, is higher $(16.45 \mathrm{eV})$ for $\mathrm{V}_{\mathrm{B}}$ comparing to that in $\mathrm{V}_{\mathrm{N}}(12.87 \mathrm{eV})$. This implies that the removal of nitrogen atom from a pure $\mathrm{h}-\mathrm{BN}$ is easier. The band structure calculations show $\mathrm{V}_{\mathrm{B}}$ as half metallic and $\mathrm{V}_{\mathrm{N}}$ as semiconductor with indirect band gap of $0.41 \mathrm{eV}$. Magnetism is induced in both the vacant systems, $2.74 \mu \mathrm{B} /$ cell for $\mathrm{V}_{\mathrm{B}}$ and $1.00 \mu \mathrm{B} /$ cell for $\mathrm{V}_{\mathrm{N}}$.

\section{FUTURE SCOPE}

The work can be further extended in order to study the charge transfer/charge density properties and the extension of sample to higher supercells. The effect of doping in the vacant system of monolayer and bilayer can be studied.

\section{AUTHOR CONTRIBUTION}

R. Karki prepared the manuscript, and participated in the whole process of manuscript revision. K. Khatri performed the simulation. K. Adhikari, N. P. Adhikari and N. Pantha were involved in the process project design, supervision of the project and discussion/review of the manuscript.

\section{REFERENCES}

[1] Xu, M.; Liang, T.; Shi, M. \& Chen, H. Graphenelike two-dimensional materials. Chemical reviews, 113(5): 3766-3798 (2013).
[2] Kittel, C.; McEuen, P. \& McEuen, P. Introduction to solid state physics, New York: Wiley, 8: 105-130 (1996).

[3] Ashcroft, N. W. \& Mermin, N. D. Solid state physics (1976).

[4] Bollmann, W. Crystal defects and crystalline interfaces. Springer Science \& Business Media (2012).

[5] Pantha, N.; Ulman, K. and Narasimhan, S. Adsorption of methane on single metal atoms supported in graphene: Role of electron back donation in binding and activation. Journal of Chemical physics, 153: 244701 (2020).

[6] Petrescu, M. I. \& Balint, M. G. Structure and properties modifications in boron nitride. Part I: Direct polymorphic transformations mechanisms. UPB Sci. Bull., Series B, 69(1): 35-42 (2007).

[7] Ciofani, G. Potential applications of boron nitride nanotubes as drug delivery systems. Expert opinion on drug delivery, 7(8): 889-893 (2010).

[8] Golberg, D.; Bando, Y.; Huang, Y.; Terao, T.; Mitome, M.; Tang, C. \& Zhi, C. Boron nitride nanotubes and nanosheets. ACS nano, 4(6): 29792993 (2010).

[9] Abtew, T. A.; Gao, W.; Gao, X.; Sun, Y. Y.; Zhang, S. B. \& Zhang, P. Theory of oxygenboron vacancy defect in cubic boron nitride: a diamond NV- isoelectronic center. Physical review letters, 113(13): 136401 (2014).

[10] Cassabois, G.; Valvin, P. \& Gil, B. Hexagonal boron nitride is an indirect bandgap semiconductor. Nature photonics, 10(4): 262-266 (2016).

[11] Song, L.; Ci, L.; Lu, H.; Sorokin, P. B.; Jin, C.; Ni, J. \& Ajayan, P. M. Large scale growth and characterization of atomic hexagonal boron nitride layers. Nano letters, 10(8): 3209-3215 (2010).

[12] Pantha, N.; Thapa, S. \& Adhikari, N. P. FirstPrinciples Study of Molecular Adsorption of Hydrogen/s on Co-Adatom Graphene. Journal of Institute of Science and Technology, 25(1): 15-23 (2020).

[13] Meyer, J. C.; Chuvilin, A.; Algara-Siller, G.; Biskupek, J. \& Kaiser, U. Selective sputtering and atomic resolution imaging of atomically thin boron nitride membranes. Nano letters, 9(7): 2683-2689 (2009).

[14] Wang, J.; Ma, F. \& Sun, M. Graphene, hexagonal boron nitride, and their heterostructures: properties and applications. RSC advances, 7(27): 16801-16822 (2017).

[15] Pantha, N.; Bissokarma, P. \& Adhikari, N. P. First-principles study of electronic and magnetic properties of nickel doped hexagonal boron nitride (h-BN). The European Physical Journal B, 93(9): 1-6 (2020). 
[16] Majety, S.; Cao, X. K.; Dahal, R.; Pantha, B. N.; Li, J.; Lin, J. Y. \& Jiang, H. X. Semiconducting hexagonal boron nitride for deep ultraviolet photonics. In Quantum Sensing and Nanophotonic Devices IX,. International Society for Optics and Photonics, 8268: 82682R (2012, January).

[17] Huang, B. \& Lee, H. Defect and impurity properties of hexagonal boron nitride: A firstprinciples calculation. Physical Review B, 86(24): 245406 (2012).

[18] Shuaibu, A.; Adeyemi, O. J.; Ushiekpan, U. R.; Olowomofe, O. G.; Akinade, B. J. \& Kafayat, O. A. First Principle Study of Structural, Elastic and Electronic Properties of Hexagonal Boron Nitride (hex-BN) Single Layer. American Journal of Condensed Matter Physics, 9(1): 1-5 (2019).

[19] Ooi, N.; Rajan, V.; Gottlieb, J.; Catherine, Y. \& Adams, J. B. Structural properties of hexagonal boron nitride. Modelling and Simulation in Materials Science and Engineering, 14(3): 515 (2006).

[20] Solozhenko, V. L.; Lazarenko, A. G.; Petitet, J. P. \& Kanaev, A. V. Bandgap energy of graphite-like hexagonal boron nitride. Journal of Physics and Chemistry of Solids, 62(7): 1331-1334 (2001).

[21] Hu, W. \& Yang, J. First-principles study of twodimensional van der Waals heterojunctions. Computational Materials Science, 112: 518-526 (2016).

[22] Wang, H.; Zhao, Y.; Xie, Y.; Ma, X. \& Zhang, X. Recent progress in synthesis of two-dimensional hexagonal boron nitride. Journal of Semiconductors, 38(3): 031003 (2017).

[23] Bockstedte, M.; Kley, A.; Neugebauer, J. \& Scheffler, M. Density-functional theory calculations for poly-atomic systems: electronic structure, static and elastic properties and ab initio molecular dynamics. Computer physics communications, 107(1-3): 187-222 (1997).
[24] Pfrommer, B. G.; Côté, M.; Louie, S. G. \& Cohen, M. L. Relaxation of crystals with the quasi-Newton method. Journal of Computational Physics, 131(1): 233-240 (1997).

[25] Jin, C.; Lin, F.; Suenaga, K. \& Iijima, S. Fabrication of a freestanding boron nitride single layer and its defect assignments. Physical review letters, 102(19): 195505 (2009).

[26] Hou, Z.; Wang, X.; Ikeda, T.; Terakura, K.; Oshima, M.; Kakimoto, M. A. \& Miyata, S. Interplay between nitrogen dopants and native point defects in graphene. Physical Review B, 85(16): 165439 (2012)

[27] Zhao, R.; Li, F.; Liu, Z.; Liu, Z. \& Ding, F. The transition metal surface passivated edges of hexagonal boron nitride (h-BN) and the mechanism of h-BN's chemical vapor deposition (CVD) growth. Physical Chemistry Chemical Physics, 17(43): 29327-29334 (2015).

[28] Martin, R. Electronic structure: basic theory and practical methods Cambridge University Press. Cambridge Daw MS, Baskes MI (1984) Phys Rev B, 296443 (2004).

[29] Kittel, C. \& McEuen, P. Introduction to solid state physics, vol 8 Wiley New York (1976).

[30] Chettri, B.; Patra, P. K.; Vu, T. V.; Nguyen, C. Q.; Yaya, A. et. al. Induced ferromagnetism in bilayer hexagonal Boron Nitride (h-BN) on vacancy defects at B and N sites. Physica E: Low-dimensional Systems and Nanostructures, 126: 114436 (2021).

[31] Barcza, G.; Ivády, V.; Szilvási, T.; Voros, M.; Veis, L. et. al. DMRG on top of plane-wave kohn-sham orbitals: a case study of defected boron nitride. Journal of Chemical Theory and Computation, 17(2): 1143-1154 (2021).

[32] Palacios, P.; Sánchez, K.; Conesa, J. C. \& Wahnón, P. First principles calculation of isolated intermediate bands formation in a transition metal-doped chalcopyrite-type semiconductor. physica status solidi (a), 203(6): 1395-1401 (2006). 